\title{
Influence of high dynamic range images on the accuracy of the photogrammetric 3D digitization: A case study
}

\author{
Santosi, Z. , , Budak, I. , , Sokac, M. ${ }^{\text {a }, ~ H a d z i s t e v i c, ~ M . ~}{ }^{a}$, Vukelic, D. ${ }^{\text {a, }}$ \\ ${ }^{a}$ University of Novi Sad, Faculty of Technical Sciences, Novi Sad, Serbia
}

\begin{abstract}
A B S T R A C T
Small and start-up companies that need product quality control can usually only afford low-cost systems. The main goal of this investigation was to estimate the influence of high dynamic range images as input for the low-cost photogrammetric structure from motion 3D digitization. Various industrial products made of metal or polymer suffer from poor visual texture. To overcome the lack of visual texture and ensure appropriate 3D reconstruction, stochastic image in the form of the light pattern was projected on the product surface. During stochastic pattern projection, a set of low dynamic range and sets of high dynamic range images were captured and processed. In this investigation digital single lens reflex camera that supports five different tonemapping operators to create high dynamic range images were used. Also, high precision measurements on a coordinate measuring machine are performed in order to verify real product geometry. The obtained results showed that reconstructed polygonal 3D models generated from high dynamic range images in this case study don't have a dominant influence on the accuracy when compared to the polygonal 3D model generated from low dynamic range images. In order to estimate 3D models dimensional accuracy, they were compared using computer-aided inspection analysis. The best achieved standard deviation distance was $+0.025 \mathrm{~mm}$ for 3D model generated based on high dynamic range images compared to the nominal CAD model.
\end{abstract}

\section{ARTICLE INFO}

Keywords:

3D digitization;

Photogrammetry;

High dynamic range (HDR) image;

Structure from motion (SfM)

*Corresponding author:

vukelic@uns.ac.rs

(Vukelic, D.)

Article history:

Received 3 June 2019

Revised 6 September 2019

Accepted 9 September 2019

\section{References}

[1] Vujica Herzog, N., Buchmeister, B., Beharic, A., Gajsek, B. (2018). Visual and optometric issues with smart glasses in Industry 4.0 working environment, Advances in Production Engineering \& Management, Vol. 13, No. 4, 417428, doi: 10.14743/apem2018.4.300.

[2] Danzl, R., Helmli, F., Scherer, S. (2011). Focus variation - A robust technology for high resolution optical 3D surface metrology, Strojniški Vestnik - Journal of Mechanical Engineering, Vol. 57, No. 3, 245-256, doi: 10.5545/svime.2010.175.

[3] Cviljušac, V., Divjak, A., Modrić, D. (2018). Computer generated holograms of 3D points cloud, Tehnički Vjesnik Technical Gazette, Vol. 25, No. 4, 1020-1027, doi: 10.17559/TV-20160726200355.

[4] Burghardt, A., Kurc, K., Szybicki, D., Muszyńska, M., Szczęch, T. (2017). Robot-operated inspection of aircraft engine turbine rotor guide vane segment geometry, Tehnički Vjesnik - Technical Gazette, Vol. 24, Supplement 2, 345-348, doi: 10.17559/TV-20160820141242.

[5] Gajic, D.B., Mihic, S., Dragan, D., Petrovic, V., Anisic, Z. (2019). Simulation of photogrammetry-based 3D data acquisition, International Journal of Simulation Modelling, Vol. 18, No. 1, 59-71, doi: 10.2507/ijsimm18(1)460.

[6] Verma, A.K., Bourke, M.C., (2019). A method based on structure-from-motion photogrammetry to generate submillimetre-resolution digital elevation models for investigating rock breakdown features, Earth Surface Dynamics, Vol. 7, No. 1, 45-66, doi: 10.5194/esurf-2018-53.

[7] Cao, M., Cao, L., Jia, W., Li, Y., Lv, Z., Zheng, L., Liu, X. (2018). Evaluation of local features for structure from motion, Multimedia Tools and Applications, Vol. 77, No. 9, 10979-10993, doi: 10.1007/s11042-018-5864-1. 
[8] Vučina, D., Bajić, D., Jozić, S., Pehnec, I. (2013). Evaluation of 3D tool wear in machining by successive stereophotogrammetry and point cloud processing, Tehnički Vjesnik - Technical Gazette, Vol. 20, No. 3, 449-458.

[9] Fraser, C.S., (2013). Automatic camera calibration in close range photogrammetry, Photogrammetric Engineering \& Remote Sensing, Vol. 79, No. 4, 381-388, doi: 10.14358/PERS.79.4.381.

[10] Hartley, R., Zisserman, A. (2004). Multiple view geometry in computer vision, Second edition, Cambridge University Press, Cambridge, United Kingdom, doi: 10.1017/CB09780511811685.

[11] Surmen, H.K., Akalan, N.E., Fetvaci, M.C., Arslan, Y.Z. (2018). A novel dorsal trimline approach for passivedynamic ankle-foot orthoses, Strojniški Vestnik - Journal of Mechanical Engineering, Vol. 64, No. 3, 185-194, doi: $10.5545 /$ sv-jme.2017.4987.

[12] Mandić, M., Galeta, T., Raos, P., Jugović, V. (2016). Dimensional accuracy of camera casing models 3D printed on Mcor IRIS: A case study, Advances in Production Engineering \& Management, Vol. 11, No. 4, 324-332, doi: 10.14743/apem2016.4.230.

[13] El-Din Fawzy, H. (2019). Study the accuracy of digital close range photogrammetry technique software as a measuring tool, Alexandria Engineering Journal, Vol. 58, No. 1, 171-179, doi: 10.1016/i.aej.2018.04.004.

[14] Percoco, G., Sánchez Salmerón, A.J. (2015). Photogrammetric measurement of 3D freeform millimetre-sized objects with micro features: An experimental validation of the close-range camera calibration model for narrow angles of view, Measurement Science and Technology, Vol. 26, No. 9, 1-9, doi: 10.1088/0957-0233/26/9/095203.

[15] Hosseininaveh Ahmadabadian, A., Karami, A., Yazdan, R. (2019). An automatic 3D reconstruction system for texture-less objects, Robotics and Autonomous Systems, Vol. 117, 29-39, doi: 10.1016/j.robot.2019.04.001.

[16] Mistry, D., Banerjee, A. (2017). Comparison of feature detection and matching approaches: SIFT and SURF, GRD Journals - Global Research and Development Journal for Engineering, Vol. 2, No. 4, 7-13.

[17] Santoši, Ž., Budak, I., Šokac, M., Puškar, T., Vukelić, Đ., Trifković, B. (2018). 3D digitization of featureless dental models using close range photogrammetry aided by noise based patterns, Facta Universitatis, Series: Mechanical Engineering, Vol. 16, No. 3, 297-305, doi: 10.22190/FUME170620029S.

[18] Lu, P.-Y., Huang, T.-H., Wu, M.-S., Cheng, Y.-T., Chuang, Y.-Y. (2009). High dynamic range image reconstruction from hand-held cameras, In: Proceedings of 2009 IEEE Conference on Computer Vision and Pattern Recognition, Miami, USA, 509-516, doi: 10.1109/CVPRW.2009.5206768.

[19] Gómez-Gutiérrez, Á., De Sanjosé-Blasco, J.J., Lozano-Parra, J., Berenguer-Sempere, F., De Matías-Bejarano, J. (2015). Does HDR pre-processing improve the accuracy of 3D models obtained by means of two conventional SfM-MVS software packages? The case of the corral del veleta rock glacier, Remote Sensing, Vol. 7, No. 8, 1026910294, doi: $10.3390 /$ rs70810269.

[20] Suma, R., Stavropoulou, G., Stathopoulou, E.K., Van Gool, L., Georgopoulos, A., Chalmers, A. (2016). Evaluation of the effectiveness of HDR tone-mapping operators for photogrammetric applications, Virtual Archaeology Review, Vol. 7, No. 15, 54-66, doi: 10.4995/var.2016.6319.

[21] Tang, C.H.H., Tang, H.E., Tay, P.K.J. (2016). Low cost digital close range photogrammetric measurement of an asbuilt anchor handling tug hull, Ocean Engineering, Vol. 119, 67-74, doi: 10.1016/j.oceaneng.2016.04.016.

[22] Cajal, C., Santolaria, J., Samper, D., Garrido, A. (2015). Simulation of laser triangulation sensors scanning for design and evaluation purposes, International Journal of Simulation Modelling, Vol. 14, No. 2, 250-264, doi: $10.2507 /$ IJSIMM14(2)6.296.

[23] Pierce, M., George, C., Dive, R., Ferguson, N. (2015). The ultimate Canon SLR handbook, Volume 4, Future Publishing Limited, United Kingdom.

[24] Eltner, A., Kaiser, A., Castillo, C., Rock, G., Neugirg, F., Abellán, A. (2016). Image-based surface reconstruction in geomorphometry - merits, limits and developments, Earth Surface Dynamics, Vol. 4, No. 2, 359-389, doi: 10.5194/esurf-4-359-2016.

[25] Bianco, S., Ciocca, G., Marelli, D. (2018). Evaluating the performance of structure from motion pipelines, Journal of Imaging, Vol. 4, No. 8, Article number: 98, doi: 10.3390/jimaging4080098.

[26] Santoši, Ž., Šokac, M., Korolija-Crkvenjakov, D., Kosec, B., Soković, M., Budak, I., (2015). Reconstruction of 3D models of cast sculptures using close-range photogrammetry, Metalurgija, Vol. 54. No. 4, 695-698.

[27] Galantucci, L.M., Percoco, G., Ferrandes, R. (2006). Accuracy issues of digital photogrammetry for 3D digitization of industrial products, Revue Internationale d'Ingénierie Numérique, Vol. 2, No. 1-2, 29-40.

[28] Page, D., Koschan, A., Voisin, S., Ali, N., Abidi, M. (2005). 3D CAD model generation of mechanical parts using coded-pattern projection and laser triangulation systems, Assembly Automation, Vol. 25, No. 3, 230-238, doi: 10.1108/01445150510610953.

[29] Agisoft LLC. Metashape - photogrammetric processing of digital images and 3D spatial data generation, from https://www.agisoft.com, accessed February 19, 2019.

[30] GOM Inspect. Software for 3D measurement data, from https://www.gom.com/3d-software/gom-inspect.html, accessed September 6, 2018.

[31] Agisoft LLC. (2018). Agisoft metashape user manual, Professional edition, Version 1.5, Agisoft LLC, St. Petersburg, Russia, from https://www.agisoft.com/pdf/metashape-pro 15 en.pdf accessed June 2, 2019.

[32] Koutsoudis, A., Vidmar, B., Ioannakis, G., Arnaoutoglou, F., Pavlidis, G., Chamzas, C. (2014). Multi-image 3D reconstruction data evaluation, Journal of Cultural Heritage, Vol. 15, No. 1, 73-79, doi: 10.1016/j.culher.2012.12.003.

[33] Baier, W., Rando, C. (2016). Developing the use of Structure-from-Motion in mass grave documentation, Forensic Science International, Vol. 261, 19-25, doi: 10.1016/i.forsciint.2015.12.008. 


\title{
Vpliv slik z visokim dinamičnim obsegom na natančnost fotogrametrične 3D digitalizacije: Študija primera
}

\author{
Santosi, Z. ${ }^{a}$, Budak, I. ${ }^{\mathrm{a}}$, Sokac, M. ${ }^{\mathrm{a}}$, Hadzistevic, M. ${ }^{\mathrm{a}}$, Vukelic, D. ${ }^{\mathrm{a},{ }^{*}}$ \\ ${ }^{a}$ University of Novi Sad, Faculty of Technical Sciences, Novi Sad, Serbia
}

\begin{abstract}
POVZETEK
Majhna in zagonska podjetja, ki rabijo nadzor kakovosti izdelkov, si običajno lahko privoščijo le poceni sisteme. Glavni cilj te raziskave je bil oceniti vpliv uporabe slik z visokim dinamičnim obsegom za poceni fotogrametrično digitalizacijo strukture iz 3D gibanja. Različni industrijski izdelki iz kovine ali polimera imajo slabo vizualno teksturo. Da bi premagali težave s teksturo in zagotovili ustrezno 3D rekonstrukcijo, je bila na površino izdelka projicirana stohastična slika v obliki svetlobnega vzorca. Med stohastično projekcijo vzorcev smo zajeli in obdelali niz slik nizkega dinamičnega obsega in niz slik visokega dinamičnega obsega. $V$ tej raziskavi smo uporabili digitalno refleksno kamero z enim objektivom, ki podpira pet različnih operatorjev za kartiranje tonov za ustvarjanje slik z visokim dinamičnim obsegom. Da se preveri realna geometrija izdelka, so bile izvedene visoko natančne meritve na koordinatnem merilnem stroju. Pridobljeni rezultati so pokazali, da rekonstruirani poligonalni 3D modeli, ustvarjeni iz slik z visokim dinamičnim obsegom, v tej študiji primera nimajo prevladujočega vpliva na točnost $\mathrm{v}$ primerjavi $\mathrm{s}$ poligonalnim 3D modelom, ustvarjenim iz slik z nizkim dinamičnim obsegom. Za oceno dimenzijske natančnosti 3D modelov je bila izvedena primerjava $\mathrm{z}$ računalniško podprto analizo. $\mathrm{V}$ primerjavi z nominalnim CAD modelom je bila najboljša razdalja s standardno deviacijo $+0.025 \mathrm{~mm}$ dosežena za 3D model, ustvarjen na podlagi slik z visokim dinamičnim obsegom.
\end{abstract}

\section{PODATKI O ČLANKU}

Ključne besede:

3D digitalizacija;

Fotogrametrija;

Slika z visokim dinamičnim obsegom (HDR);

Struktura iz gibanja (SfM)

*Kontaktna oseba: vukelic@uns.ac.rs (Vukelic, D.)

Zgodovina članka: Prejet 3. junija 2019

Popravljen 6. septembra 2019

Sprejet 9. septembra 2019 